\title{
Depressor and Anti-Inflammatory Effects of Angiotensin II Receptor Blockers in Metabolic and/or Hypertensive Patients With Coronary Artery Disease: A Randomized, Prospective Study (DIAMOND Study)
}

\author{
Sen Adachi ${ }^{a}$, Shin-ichiro Miura ${ }^{a}$, b, c, Yuhei Shiga ${ }^{a}$, Tadaaki Arimura ${ }^{a}$, Takashi Kuwano ${ }^{a}$, Ken Kitajima ${ }^{a}$, \\ Amane Ike $^{a}$, Makoto Sugihara ${ }^{\mathrm{a}}$, Atsushi Iwata ${ }^{\mathrm{a}}$, Hiroaki Nishikawa ${ }^{\mathrm{a}}$, Natsumi Morito ${ }^{\mathrm{a}}$, Keijiro Saku ${ }^{\mathrm{a}, \mathrm{b}}$
}

\begin{abstract}
Background: We compared the efficacy and safety of azilsartan to those of olmesartan in a prospective, randomized clinical trial.

Methods: Forty-four hypertensive patients who had coronary artery disease (CAD) were enrolled. We randomly assigned patients to changeover from their prior angiotensin II receptor blockers (ARBs) to either azilsartan or olmesartan, and followed the patients for 12 weeks.

Results: Office systolic blood pressure (SBP) in the azilsartan group was significantly decreased after 12 weeks. SBP and diastolic blood pressure (DBP) after 12 weeks in the azilsartan group were significantly lower than those in the olmesartan group. The percentage of patients who reached the target BP at 12 weeks $(78 \%)$ in the azilsartan group was significantly higher than that at 12 weeks $(45 \%)$ in the olmesartan group. There were no significant changes in pentraxin-3, high-sensitively C-reactive protein or adiponectin in blood after 12 weeks in either group. Although serum levels of creatinine $(\mathrm{Cr})$ in the azilsartan group significantly increased, these changes were within the respective normal range.
\end{abstract}

Conclusion: In conclusion, the ability of azilsartan to reduce BP may be superior to that of prior ARBs with equivalent safety in hypertensive patients with $\mathrm{CAD}$.

Keywords: Blood pressure; Angiotensin II type 1 receptor blocker; Creatinine

Manuscript accepted for publication August 05, 2016

aDepartment of Cardiology, Fukuoka University School of Medicine, Fukuoka 814-0180, Japan

bDepartment of Molecular Cardiovascular Therapeutics, Fukuoka University School of Medicine, Fukuoka 814-0180, Japan

${ }^{\mathrm{c} C}$ Corresponding Author: Shin-ichiro Miura, Department of Cardiology, Fukuoka University School of Medicine, 7-45-1 Nanakuma, Jonan-ku, Fukuoka 814-0180, Japan.Email: miuras@cis.fukuoka-u.ac.jp

doi: http://dx.doi.org/10.14740/jocmr2675w

\section{Introduction}

Seven angiotensin II type $1\left(\mathrm{AT}_{1}\right)$ receptor blockers (ARBs) are available for clinical use in Japan [1,2]. We have previously compared the efficacy and safety of valsartan and losar$\tan [3,4]$ and of irbesartan and olmesartan $[5,6]$ in patients with hypertension (HTN). All ARBs may not have the same depressor effects. We have proposed that small differences in the molecular structures of ARBs could lead to differences in their abilities to influence the $\mathrm{AT}_{1}$ receptor (molecule-specific (differential) effects) [2, 7].

Azilsartan medoxomil and azilsartan have been reported to have greater antihypertensive effects than other ARBs [813]. The results of comparisons of the beneficial effects of azilsartan and other ARBs were not consistent. For example, azilsartan medoxomil lowered systolic blood pressure (SBP) by a greater magnitude than olmesartan or valsartan at maximally approved doses in patients with prediabetes mellitus and type 2 diabetes mellitus (DM) [12]. On the other hand, olmesartan reduced angiotensin II and aldosterone levels and left ventricular mass index more effectively than azilsartan in hypertensive outpatients who were clinically stable after cardiac surgery [13].

The purpose of this study was to confirm which of the ARBs, azilsartan and olmesartan, has a superior depressor effect in patients with HTN. In this study, we enrolled hypertensive patients with coronary artery disease (CAD) who changed over from their prior ARBs to azilsartan or olmesartan, and compared the efficacy and safety of azilsartan to those of olmesartan. In particular, we analyzed whether anti-inflammation and inflammation markers (adiponectin, pentraxin-3 (PTX-3) and high-sensitive $\mathrm{C}$-reactive protein (hs-CRP)) were associated with the reduction of BP by ARBs.

\section{Methods}

\section{Patients}

The participants included 44 hypertensive patients with CAD who were being treated with ARBs other than azilsartan and 
Table 1. Patient Characteristic at Baseline in the Azilsartan and Olmesartan Groups

\begin{tabular}{|c|c|c|c|}
\hline & Azilsartan group $(\mathrm{n}=18)$ & Olmesartan group $(n=20)$ & $P$ value (azilsartan vs. olmesartan) \\
\hline Age (years) & $68 \pm 7$ & $71 \pm 9$ & 0.24 \\
\hline Male (\%) & 78 & 65 & 0.40 \\
\hline BMI $\left(\mathrm{kg} / \mathrm{m}^{2}\right)$ & $26.1 \pm 2.7$ & $26.0 \pm 3.7$ & 0.98 \\
\hline $\mathrm{WC}(\mathrm{cm})$ & $94 \pm 7$ & $90 \pm 11$ & 0.18 \\
\hline MetS (\%) & 89 & 65 & 0.09 \\
\hline Smoking (\%) & 72 & 50 & 0.17 \\
\hline DM $(\%)$ & 50 & 55 & 0.76 \\
\hline DL $(\%)$ & 94 & 95 & 0.94 \\
\hline HU $(\%)$ & 17 & 30 & 0.35 \\
\hline SBP (mm Hg) & $128 \pm 17$ & $129 \pm 11$ & 0.99 \\
\hline DBP (mm Hg) & $71 \pm 14$ & $70 \pm 12$ & 0.93 \\
\hline PR (/min) & $67 \pm 10$ & $72 \pm 11$ & 0.18 \\
\hline LVEF (\%) & $63 \pm 11$ & $62 \pm 9$ & 0.88 \\
\hline \multicolumn{4}{|l|}{ Medication } \\
\hline$\beta$-blocker (\%) & 44 & 20 & 0.11 \\
\hline$\alpha$-blocker (\%) & 28 & 10 & 0.17 \\
\hline CCB $(\%)$ & 50 & 70 & 0.22 \\
\hline Diuretics (\%) & 0 & 20 & 0.046 \\
\hline Statin (\%) & 89 & 80 & 0.47 \\
\hline Nicorandil (\%) & 22 & 10 & 0.32 \\
\hline SU (\%) & 6 & 5 & 0.94 \\
\hline Metformin (\%) & 6 & 20 & 0.20 \\
\hline DPP4-I (\%) & 39 & 40 & 0.95 \\
\hline Insulin (\%) & 22 & 15 & 0.35 \\
\hline
\end{tabular}

BMI: body mass index; WC: waist circumference; MetS: metabolic syndrome; DM: diabetes mellitus; DL: dyslipidemia; HU: hyperuricemia; SBP: systolic blood pressure; DBP: diastolic BP; PR: pulse rate; LVEF: left ventricular ejection fraction; CCB: calcium channel blocker; SU: sulfonylurea; DPP4-I: dipeptidyl peptidase-4 inhibitor.

olmesartan. All patients underwent coronary angiography $(\mathrm{CAG})$. When the patients had a significant coronary stenosis, they underwent successful percutaneous coronary intervention. We randomly assigned patients to changeover from their prior ARBs to either azilsartan or olmesartan, and followed the patients for 12 weeks. Although combined treatment with other antihypertensive drugs was permitted, the doses of these medications were not changed and other antihypertensive drugs were not added throughout the study period. We excluded patients with secondary HTN, acute coronary syndrome within the previous 6 months, pregnancy, a history of allergy to azilsartan or olmesartan, familial hypercholesterolemia, severe liver dysfunction, severe kidney dysfunction, malignancy, collagen disease, or steroid use. In addition, six patients withdrew because they had hypotension $(\mathrm{n}=1)$, did not visit the hospital $(\mathrm{n}=4)$ or committed a protocol violation $(\mathrm{n}=1)$ during the study period. Therefore, we finally analyzed 38 hypertensive patients (18 and 20 in the azilsartan and olmesartan groups, respectively). The primary endpoint was the depressor effect of BP in the azilsartan and olmesartan groups. Secondary endpoints were the changes in biochemical parameters in blood between baseline and after 12 weeks in the two groups. The protocol in this study was approved by the Ethics Committee of Fukuoka University Hospital (\#12-10-01) and registered under UMIN000016247. All of the subjects gave their written informed consent to participate.

\section{BP and biochemical parameter measurements}

BP was measured in the right arm after a 5-min rest period, and the average of three consecutive BP measurements was used. Patients who were under antihypertensive treatment were considered to have HTN. Blood samples were collected following an overnight fast using standard techniques. We examined biochemical parameters in blood, including urea nitrogen, creatinine $(\mathrm{Cr})$, estimated glomerular filtration rate (GFR), uric acid, sodium, potassium, chloride, low-density 

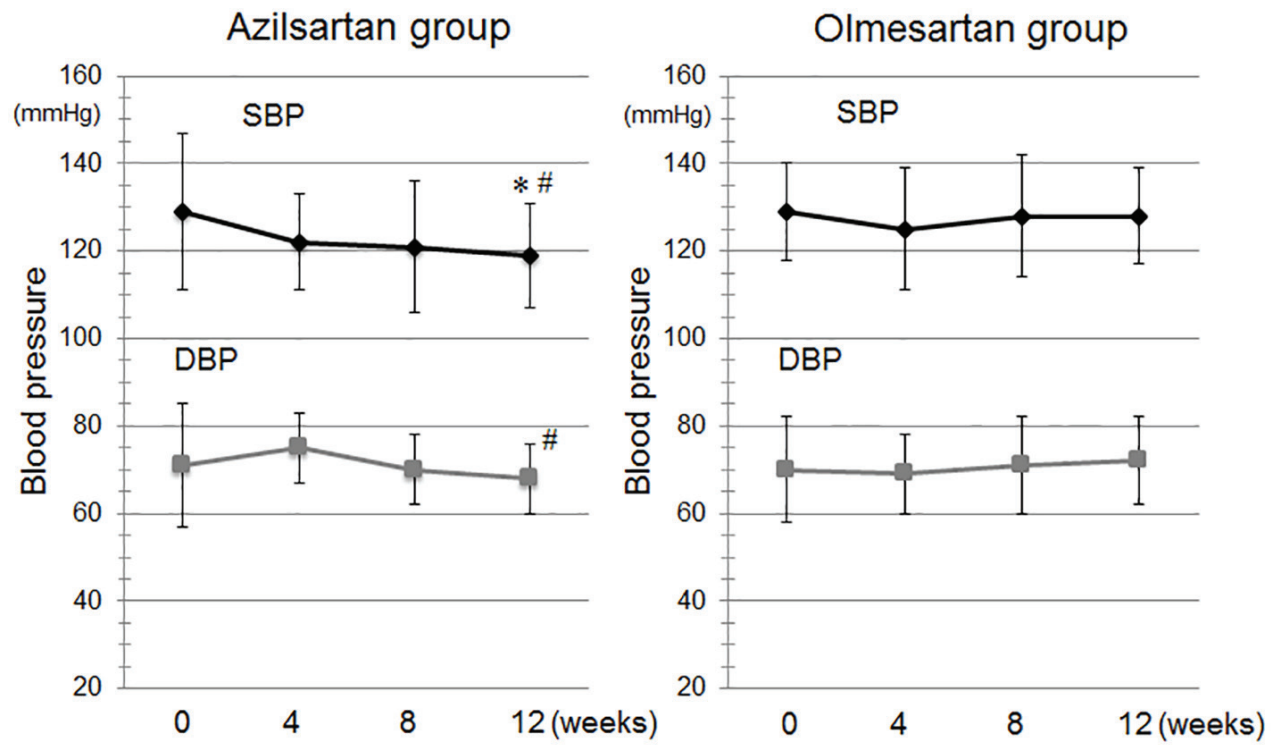

Figure 1. Time course of systolic blood pressure (SBP) and diastolic BP (DBP) during the study period in the azilsartan and olmesartan groups. ${ }^{*} \mathrm{P}<0.05$ vs. at baseline in the azilsartan group. \#P $<0.05$ vs. at 12 weeks in the olmesartan group.

lipoprotein cholesterol (LDL-C), high-density lipoprotein cholesterol (HDL-C), triglycerides (TG), total cholesterol, aspartate aminotransferase, alanine aminotransferase, lactate dehydrogenase, creatinine kinase, glucose, hemoglobin A1c, $\mathrm{N}$-terminal pro-brain natriuretic peptide, adiponectin, PTX-3 and hs-CRP. The concentrations of adiponectin and PTX-3 in plasma were determined in duplicate using specific enzyme immunoassays according to the manufacturer's instructions (R\&D Systems, Minneapolis, MN, and Perseus Proteomics Inc., Tokyo, Japan). At our laboratory, the intra- and inter-assay coefficients of variation for these parameters were each $<$ $5 \%$. Patients with an LDL-C level $\geq 140 \mathrm{mg} / \mathrm{dL}$, a TG level $\geq$ $150 \mathrm{mg} / \mathrm{dL}$ or an HDL-C level $<40 \mathrm{mg} / \mathrm{dL}$ or who were being treated for dyslipidemia (DL) were diagnosed with DL. Patients who were being treated for DM or who had symptoms of DM and a fasting blood glucose concentration $\geq 126 \mathrm{mg} /$ $\mathrm{dL}$ were considered to have DM. Otherwise, the results of a 75-g oral glucose tolerance test were used to diagnose DM. A diagnosis of metabolic syndrome (MetS) was based on body mass index $(\mathrm{BMI}) \geq 25 \mathrm{~kg} / \mathrm{m}^{2}$ and at least two of the following criteria: $\mathrm{SBP} \geq 130 \mathrm{~mm} \mathrm{Hg}$ and/or diastolic BP (DBP) $\geq 85 \mathrm{~mm} \mathrm{Hg}$, HDL-C $<40 \mathrm{mg} / \mathrm{dL}$ and/or $\mathrm{TG} \geq 150 \mathrm{mg} / \mathrm{dL}$, and fasting blood sugar $>110 \mathrm{mg} / \mathrm{dL}$. BMI was calculated as weight $(\mathrm{kg}) /$ height $(\mathrm{m})^{2}$.

\section{Statistical analysis}

The statistical analysis was performed using the Stat View statistical software package (Stat View 5; SAS Institute Inc., Cary, $\mathrm{NC}$, USA). The data are shown as the mean \pm standard deviation. Categorical variables were compared between groups using a Chi-square analysis or Fisher's exact test. The differences in continuous variables between groups were examined using Student's $t$-test or Wilcoxon's rank-sum test. Differences were considered to be statistically significant when the P values were $<0.05$.

\section{Results}

\section{Patient characteristics at baseline}

Table 1 shows the patient characteristics at baseline. Before the study, the patients were receiving ARBs such as telmisartan (n $=6,40 \mathrm{mg} /$ day $)$, losartan $(\mathrm{n}=11,53 \pm 25 \mathrm{mg} /$ day $)$, candesar$\tan (\mathrm{n}=4,7.0 \pm 1.7 \mathrm{mg} /$ day $)$, valsartan $(\mathrm{n}=13,63 \pm 35 \mathrm{mg} /$ day) or irbesartan ( $\mathrm{n}=4,88 \pm 22 \mathrm{mg} /$ day $)$. The average doses of ARBs in the azilsartan and olmesartan groups were $27 \pm 11$ and $23 \pm 9 \mathrm{mg} /$ day, respectively, and this difference was not significant $(\mathrm{P}=0.23)$. There were also no differences in several coronary risk factors, such as smoking, DM, DL and gender. There were no significant differences in the use of medications such as $\beta$-blockers, calcium channel blockers, statins or nicorandil, except for diuretics, between the groups.

\section{Time courses of $B P$ and pulse rate (PR) during the study period}

Figure 1 shows the time course of BP during the study period. There were no differences in BP at baseline between the azilsartan and olmesartan groups. SBP was significantly reduced at 12 weeks in the azilsartan group. BP was not significantly decreased in patients who received a changeover to olmesartan. In addition, SBP and DBP after 12 weeks in the azilsartan group were significantly lower than those in the olmesartan group. There were no changes in PR throughout the study period (data not shown). 


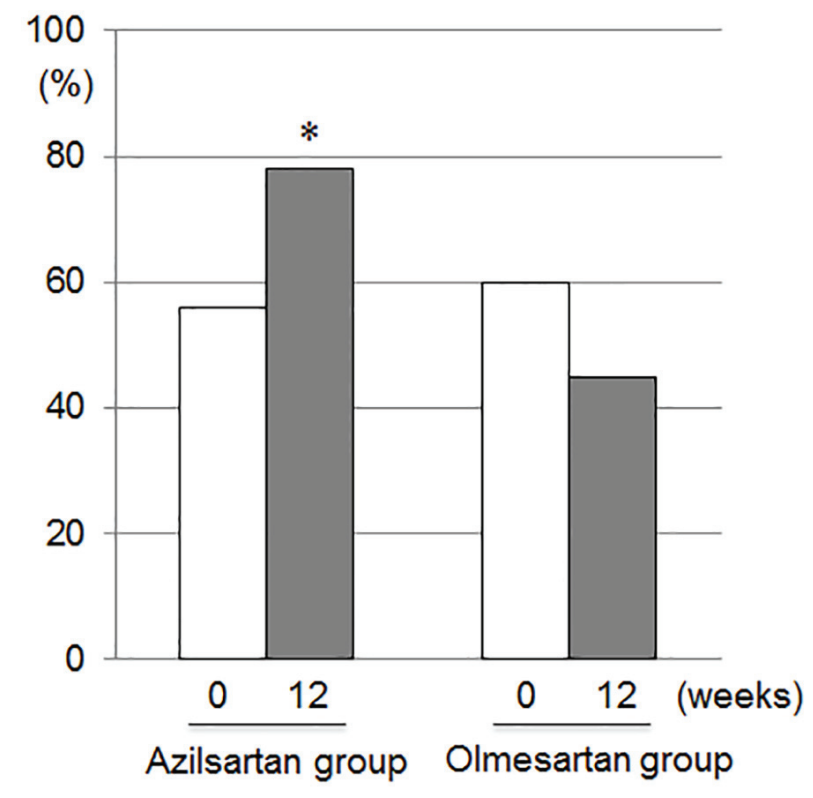

Figure 2. Percentages of patients who reached the target blood pressure $(<130 / 80 \mathrm{~mm} \mathrm{Hg})$ at 0 and 12 weeks in the azilsartan and olmesartan groups. ${ }^{*} \mathrm{P}<0.05$ vs. at 12 weeks in the olmesartan group

\section{Percentages (\%) of patients who reached the target BP at 0 and 12 weeks in the azilsartan and olmesartan groups}

Since 15 patients who had a significant coronary artery stenosis underwent successful percutaneous coronary intervention and 23 patients did not have the significant coronary stenosis in this study, a desirable target BP in the absence of significant coronary stenosis is $<130 / 80 \mathrm{~mm} \mathrm{Hg}$ [14]. There was no significant difference in percentage of patients who reached the target $\mathrm{BP}(<130 / 80 \mathrm{~mm} \mathrm{Hg})$ at 0 weeks between the azilsartan and olmesartan groups (Fig. 2). The percentage of patients who reached the target BP at 12 weeks (78\%) in the azilsartan group was significantly higher than that at 12 weeks $(45 \%)$ in the olmesartan group.

\section{Biochemical parameters in blood at 0 and 12 weeks}

The biochemical parameters in blood at 0 and 12 weeks are shown in Table 2. All parameters at baseline were similar between the azilsartan and olmesartan groups. Serum levels of $\mathrm{Cr}$ in the azilsartan group significantly increased after 12 weeks. Figure 3 shows blood concentrations of inflammation and anti-inflammation markers such as adi-

Table 2. The Biochemical Parameters in Blood at 0 and 12 Weeks in the Azilsartan and Olmesartan Groups

\begin{tabular}{|c|c|c|c|c|c|c|}
\hline & \multicolumn{3}{|c|}{ Azilsartan group ( $\mathrm{n}=18)$} & \multicolumn{3}{|c|}{ Olmesartan group $(n=20)$} \\
\hline & 0 weeks & 12 weeks & $\begin{array}{l}\text { P value } \\
(0 \text { vs. } 12 \text { weeks) }\end{array}$ & 0 weeks & 12 weeks & $\begin{array}{l}\text { P value } \\
(0 \text { vs. } 12 \text { weeks) }\end{array}$ \\
\hline $\mathrm{UN}, \mathrm{mg} / \mathrm{dL}$ & $17 \pm 5$ & $18 \pm 5$ & 0.38 & $19 \pm 6$ & $20 \pm 9$ & 0.50 \\
\hline $\mathrm{Cr}, \mathrm{mg} / \mathrm{dL}$ & $0.9 \pm 0.2$ & $1.0 \pm 0.2$ & 0.009 & $1.0 \pm 0.3$ & $1.0 \pm 0.2$ & 0.08 \\
\hline $\mathrm{eGFR}, \mathrm{mL} / \mathrm{min} / 1.73 \mathrm{~m}^{2}$ & $63 \pm 13$ & $58 \pm 12$ & 0.003 & $58 \pm 17$ & $55 \pm 15$ & 0.55 \\
\hline $\mathrm{UA}, \mathrm{mg} / \mathrm{dL}$ & $5.5 \pm 1.1$ & $5.7 \pm 0.8$ & 0.67 & $5.2 \pm 1.1$ & $5.9 \pm 1.6$ & 0.17 \\
\hline $\mathrm{Na}, \mathrm{mEq} / \mathrm{L}$ & $142 \pm 2$ & $142 \pm 2$ & 0.67 & $141 \pm 3$ & $142 \pm 3$ & 0.64 \\
\hline $\mathrm{K}, \mathrm{mEq} / \mathrm{L}$ & $4.3 \pm 0.4$ & $4.5 \pm 0.5$ & 0.07 & $4.3 \pm 0.4$ & $4.5 \pm 0.4$ & 0.30 \\
\hline $\mathrm{Cl}, \mathrm{mEq} / \mathrm{L}$ & $105 \pm 3$ & $106 \pm 3$ & 0.12 & $106 \pm 4$ & $106 \pm 4$ & 0.70 \\
\hline LDL-C, mg/dL & $85 \pm 27$ & $87 \pm 38$ & 0.70 & $76 \pm 28$ & $77 \pm 23$ & 0.86 \\
\hline HDL-C, mg/dL & $47 \pm 15$ & $47 \pm 12$ & 0.80 & $43 \pm 13$ & $45 \pm 15$ & 0.10 \\
\hline $\mathrm{TG}, \mathrm{mg} / \mathrm{dL}$ & $150 \pm 71$ & $144 \pm 58$ & 0.70 & $144 \pm 86$ & $139 \pm 82$ & 0.69 \\
\hline $\mathrm{TC}, \mathrm{mg} / \mathrm{dL}$ & $160 \pm 23$ & $166 \pm 42$ & 0.55 & $146 \pm 33$ & $148 \pm 30$ & 0.64 \\
\hline AST, IU/L & $27 \pm 7$ & $27 \pm 7$ & 0.96 & $27 \pm 14$ & $26 \pm 12$ & 0.60 \\
\hline ALT, IU/L & $26 \pm 9$ & $27 \pm 12$ & 0.72 & $26 \pm 14$ & $24 \pm 10$ & 0.15 \\
\hline LDH, IU/L & $202 \pm 36$ & $195 \pm 28$ & 0.39 & $197 \pm 35$ & $186 \pm 31$ & 0.08 \\
\hline $\mathrm{CK}, \mathrm{IU} / \mathrm{L}$ & $132 \pm 79$ & $128 \pm 58$ & 0.80 & $117 \pm 73$ & $116 \pm 68$ & 0.97 \\
\hline Glu, mg/dL & $115 \pm 30$ & $124 \pm 60$ & 0.56 & $121 \pm 30$ & $126 \pm 38$ & 0.51 \\
\hline HbA1c, \% & $6.6 \pm 1.0$ & $6.8 \pm 1.3$ & 0.22 & $7.0 \pm 1.7$ & $6.7 \pm 1.5$ & 0.36 \\
\hline NT-proBNP, pg/mL & $114 \pm 96$ & $111 \pm 104$ & 0.90 & $324 \pm 504$ & $203 \pm 258$ & 0.07 \\
\hline
\end{tabular}

UN: urea nitrogen; Cr: creatinine; eGFR: estimated glomerular filtration rate; UA: uric acid; Na: sodium; K: potassium; Cl: chloride; LDL-C: low-density lipoprotein cholesterol; HDL-C: high-density lipoprotein cholesterol; TG: triglycerides; TC: total cholesterol; AST: aspartate aminotransferase; ALT: alanine aminotransferase; LDH: lactate dehydrogenase; CK: creatinine kinase; Glu: glucose; HbA1c: hemoglobin A1c; NT-proBNP: N-terminal pro-brain natriuretic peptide. 


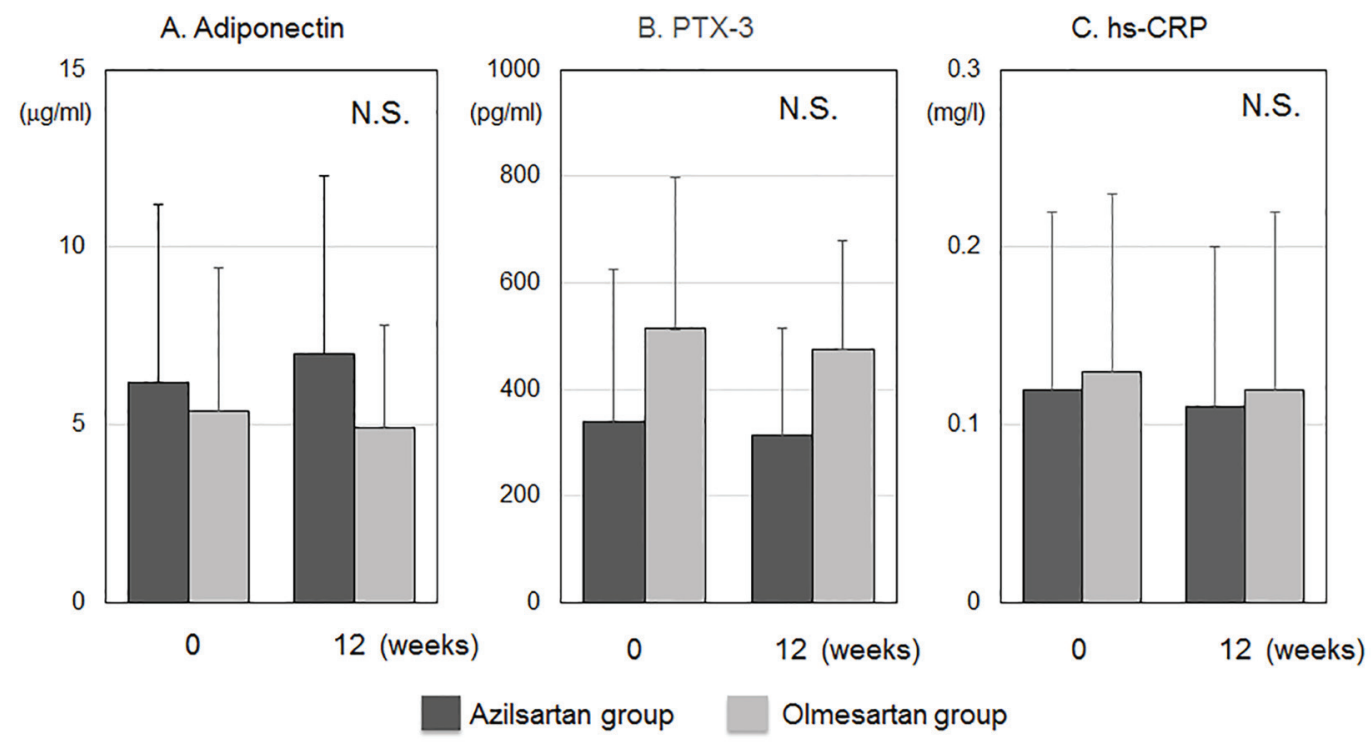

Figure 3. Blood concentrations of anti-inflammation and inflammation markers such as adiponectin (A), pentraxin-3 (PTX-3) (B) and high-sensitive C-reactive protein (hs-CRP) (C). N.S., not significant.

ponectin, PTX-3 and hs-CRP. All markers at baseline and after 12 weeks were similar between the azilsartan and olmesartan groups.

\section{Discussion}

In the present study, the depressor effect in the azilsartan group may be superior to that in the olmesartan group, and there were no serious side effects under treatment with azilsartan or olmesartan, although $\mathrm{Cr}$ levels in the azilsartan group significantly increased.

Cardiovascular and renal protections are needed to maintain optimal BP [15-17]. A 10\% lower incidence of stroke mortality and a 7\% lower incidence of mortality from CAD or other vascular causes were associated with a $2 \mathrm{~mm} \mathrm{Hg}$ reduction in SBP in middle-aged subjects [18]. Therefore, the reduction in SBP of about $8 \mathrm{~mm} \mathrm{Hg}$ with azilsartan in this study may provide clinical benefits. In addition, percentage of patients who reached the target BP at 12 weeks was $78 \%$ in the azilsartan group, whereas percentage of patients at 12 weeks was $45 \%$ in the olmesartan group.

Although serum $\mathrm{Cr}$ levels in the azilsartan group significantly increased after 12 weeks, the changes in $\mathrm{Cr}$ were within the respective normal ranges. In addition, none of the patients received new medications or changed the doses of their medications during the 12 -week study period. This is a usual observation because $\mathrm{Cr}$ increases shortly after the initiation of treatment with inhibitors of the renin-angiotensin system (RAS) (ARB or angiotensin converting enzyme inhibitor (ACE-I)) or with a change to a stronger ARB or ACE-I. This phenomenon is functionally important in nature due to the adaptive effect in response to a reduction in glomerular BP and its dependence on the RAS to maintain GFR [19]. Thus, the slight increase in $\mathrm{Cr}$ in this study should not cause concern.

The differences between ARBs have been observed as pleiotropic effects, in addition to the ability of these drugs to lower BP [1,2]. Blood concentrations of inflammation and anti-inflammation markers such as hs-CRP, PTX-3 and adiponectin did not change in either the azilsartan or olmesartan group. We previously reported that reductions in coronary restenosis and anti-inflammation after percutaneous coronary intervention were observed under ARB treatment [3]. This discrepancy may be due to the wide variation in the respective markers among patients.

This study has two important limitations. First, the sample size is relatively small, which limits our ability to determine significance. Second, we applied a changeover, where we switched from various ARBs to azilsartan or olmesartan. A crossover study would be preferable. However, the patients were divided into the two groups randomly, and this may have minimized any difference in BP.

In conclusion, the depressor effect of azilsartan may be superior to that of prior ARBs, and there were no serious changes in biochemical parameters under treatment with azilsartan or olmesartan, although the level of $\mathrm{Cr}$ increased in patients treated with azilsartan.

\section{Conflicts of Interest}

K.S. is a Chief Director and S.M. is a Director of NPO Clinical and Applied Science, Fukuoka, Japan. K.S. has an Endowed "Department of Molecular Cardiovascular Therapeutics" supported by MSD, Co. Ltd. S.M. belongs to the Department of Molecular Cardiovascular Therapeutics, which is supported by MSD, Co. Ltd. K.S and S.M. received research grants and lecture honoraria from Takeda Pharm Co. Ltd. and Daiichi Sankyo Co. Ltd. 


\section{References}

1. Imaizumi S, Miura S, Yahiro E, Uehara Y, Komuro I, Saku K. Class- and molecule-specific differential effects of angiotensin II type 1 receptor blockers. Curr Pharm Des. 2013;19(17):3002-3008.

2. Miura S, Karnik SS, Saku K. Review: angiotensin II type 1 receptor blockers: class effects versus molecular effects. J Renin Angiotensin Aldosterone Syst. 2011;12(1):1-7.

3. Iwata A, Miura S, Imaizumi S, Kiya $Y$, Nishikawa $H$, Zhang B, Shimomura H, et al. Do valsartan and losartan have the same effects in the treatment of coronary artery disease? Circ J. 2007;71(1):32-38.

4. Shiga Y, Miura S, Morii J, Kuwano T, Mitsutake R, Uehara $\mathrm{Y}$, Inoue $\mathrm{A}$, et al. Comparison of the efficacy and safety of single-pill fixed-dose combinations of losartan/ hydrochlorothiazide and valsartan/hydrochlorothiazide in patients with hypertension (SALT-VAT study). Intern Med. 2011;50(21):2477-2483.

5. Morii J, Miura S, Shiga Y, Sugihara M, Arimura T, Sako $\mathrm{H}$, Zhang B, et al. Comparison of the efficacy and safety of irbesartan and olmesartan in patients with hypertension (EARTH study). Clin Exp Hypertens. 2012;34(5):342349.

6. Morii J, Miura S, Ike A, Shiga Y, Sugihara M, Iwata A, Kawamura A, et al. Comparison of the efficacies of irbesartan and olmesartan after successful coronary stent implantation. Intern Med. 2013;52(7):713-719.

7. Fujino M, Miura S, Kiya Y, Tominaga Y, Matsuo Y, Karnik SS, Saku K. A small difference in the molecular structure of angiotensin II receptor blockers induces AT(1) receptor-dependent and -independent beneficial effects. Hypertens Res. 2010;33(10):1044-1052.

8. Rakugi H, Enya K, Sugiura K, Ikeda Y. Comparison of the efficacy and safety of azilsartan with that of candesartan cilexetil in Japanese patients with grade I-II essential hypertension: a randomized, double-blind clinical study. Hypertens Res. 2012;35(5):552-558.

9. White WB, Weber MA, Sica D, Bakris GL, Perez A, Cao C, Kupfer S. Effects of the angiotensin receptor blocker azilsartan medoxomil versus olmesartan and valsartan on ambulatory and clinic blood pressure in patients with stages 1 and 2 hypertension. Hypertension. 2011;57(3):413420.

10. Bakris GL, Sica D, Weber M, White WB, Roberts A, Perez
A, Cao C, et al. The comparative effects of azilsartan medoxomil and olmesartan on ambulatory and clinic blood pressure. J Clin Hypertens (Greenwich). 2011;13(2):8188.

11. Sica D, White WB, Weber MA, Bakris GL, Perez A, Cao $\mathrm{C}$, Handley A, et al. Comparison of the novel angiotensin II receptor blocker azilsartan medoxomil vs valsartan by ambulatory blood pressure monitoring. J Clin Hypertens (Greenwich). 2011;13(7):467-472.

12. White WB, Cuadra RH, Lloyd E, Bakris GL, Kupfer S. Effects of azilsartan medoxomil compared with olmesartan and valsartan on ambulatory and clinic blood pressure in patients with type 2 diabetes and prediabetes. J Hypertens. 2016;34(4):788-797.

13. Sezai A, Osaka S, Yaoita H, Arimoto M, Hata H, Shiono M, Sakino H. Changeover Trial of Azilsartan and Olmesartan Comparing Effects on the Renin-AngiotensinAldosterone System in Patients with Essential Hypertension after Cardiac Surgery (CHAOS Study). Ann Thorac Cardiovasc Surg. 2016;22(3):161-167.

14. Shimamoto K, Ando K, Fujita T, Hasebe N, Higaki J, Horiuchi M, Imai Y, et al. The Japanese Society of Hypertension Guidelines for the Management of Hypertension (JSH 2014). Hypertens Res. 2014;37(4):253-390.

15. Major outcomes in high-risk hypertensive patients randomized to angiotensin-converting enzyme inhibitor or calcium channel blocker vs diuretic: The Antihypertensive and Lipid-Lowering Treatment to Prevent Heart Attack Trial (ALLHAT). JAMA. 2002;288(23):29812997.

16. Staessen JA, Wang JG, Thijs L. Cardiovascular protection and blood pressure reduction: a meta-analysis. Lancet. 2001;358(9290):1305-1315.

17. Yamagata K, Ishida K, Sairenchi T, Takahashi H, Ohba S, Shiigai T, Narita M, et al. Risk factors for chronic kidney disease in a community-based population: a 10-year follow-up study. Kidney Int. 2007;71(2):159-166.

18. Lewington S, Clarke R, Qizilbash N, Peto R, Collins R. Age-specific relevance of usual blood pressure to vascular mortality: a meta-analysis of individual data for one million adults in 61 prospective studies. Lancet. 2002;360(9349):1903-1913.

19. Epstein BJ. Elevations in serum creatinine concentration: concerning or reassuring? Pharmacotherapy. 2004;24(5):697-702; discussion 702-693. 